Itinéraires Itinéraires

Littérature, textes, cultures

\title{
« Détruire » disent-ils... Violence et mystique dans Thirteen Cents et Les Villes assassines
}

Destroy, they said... Violence and Mystic in Thirteen Cents and Les Villes assassines

\section{Tina Harpin}

\section{(2) OpenEdition}

\section{Journals}

Édition électronique

URL : http://journals.openedition.org/itineraires/3294

DOI : 10.4000/itineraires.3294

ISSN : 2427-920X

Éditeur

Pléiade

\section{Référence électronique}

Tina Harpin, « « Détruire » disent-ils... Violence et mystique dans Thirteen Cents et Les Villes assassines », Itinéraires [En ligne], 2016-1 | 2016, mis en ligne le 01 décembre 2016, consulté le 30 avril 2019. URL : http://journals.openedition.org/itineraires/3294; DOI : 10.4000/itineraires.3294

Ce document a été généré automatiquement le 30 avril 2019.

\section{$\circledast \oplus \Theta \Theta$}

Itinéraires est mis à disposition selon les termes de la licence Creative Commons Attribution - Pas d'Utilisation Commerciale - Pas de Modification 4.0 International. 


\title{
« Détruire » disent-ils... Violence et mystique dans Thirteen Cents et Les Villes assassines
}

\author{
Destroy, they said... Violence and Mystic in Thirteen Cents and Les Villes \\ assassines
}

Tina Harpin

1 Les mutations qu'ont connues les métropoles urbaines depuis la fin du $\mathrm{xx}^{\mathrm{e}}$ siècle se sont accompagnées d'une production romanesque intense et variée où la ville apparaît volontiers comme un espace multiple, ludique, cosmopolite, où s'invente le quotidien et où se rêve parfois un futur utopique. La ville «muse» serait pour les écrivains, les intellectuels, les citoyens et les citadins, le lieu de réflexion privilégié d'un cosmopolitisme renouvelé (Appiah [2006] 2008, Appadurai [1996] 2001) contre les fermetures ethnocentriques et xénophobes et contre les exploitations économiques et les inquiétudes nées de l'univers du risque engendré par le capitalisme libéral. Cette vision optimiste de la ville est vivace en Afrique du Sud, alors que, paradoxalement, ce pays encore marqué géographiquement et historiquement par l'apartheid a de nombreux défis à relever en matière de politique urbaine, de justice et d'égalité (Demissie 2007, Abramson 2009). Serait-ce parce que les luttes de libération ont prouvé leur efficacité jusque dans les espaces urbains ségrégués qu'une telle vision est permise ? Depuis la « métropole » de Johannesburg, Achille Mbembe fait l'éloge d'un nouvel humanisme né des villes: l'« afropolitanisme ». Il le définit comme « ce corps en mouvement, jamais à sa place, dont le centre se déplace partout; ce corps mouvant dans l'énorme machine du monde » et dont « l'Afrique du Sud [serait] le laboratoire privilégié [...]» (Mbembe $2010: 143$ ). Le « monde-africain-qui-vient » pressenti par Achille Mbembe lui serait inspiré par une vision confiante du présent et du devenir de la ville africaine contre les plaintes de l'«afropessimisme » (Mbembe et Nuttall 2004).

2 Bien qu'Achille Mbembe souligne que « l'afropolitanisme n'est pas la même chose que le panafricanisme ou la Négritude ${ }^{1}$ ", il est clair qu'à l'instar de ces notions, le concept a 
vocation à exprimer un phénomène ${ }^{2}$ qui prend sa source en Afrique tout en faisant fi de ses frontières. Les chercheurs de l'Association pour l'étude des littératures africaines l'ont compris lorsqu'ils notent que le mot est inspiré du terme "cosmopolitisme» ( cosmopolitanism en anglais) et qu'il « met l'accent, non plus sur l'affirmation d'un "monde" africain, mais sur la circulation des mondes au sein et à partir du continent africain ${ }^{3}$ ». En ce sens, l'afropolitanisme est une notion certes attachée à un espace précis mais c'est aussi un concept voyageur, qui vise une universalité, et qui manifeste un enthousiasme rêveur quant au potentiel créateur des villes postcoloniales, laboratoires de nouveaux cosmopolitismes vernaculaires. Cette vision utopiste afropolitaine du rôle des villes postcoloniales connaît un certain succès malgré les débats qu'elle suscite (Awondo 2014), ce qui nous encourage à choisir un corpus littéraire qui en considère les limites, pour étudier leur poétique pessimiste.

Il est impossible de nier que l'ère récente de révolutions urbaines est caractérisée par la prolifération des «non-lieux » (Augé 1992) et par l'extension de la pauvreté, du chômage et des habitats précaires ${ }^{4}$ (Davis 2006). Comment donc contribuer à une réflexion sur le potentiel créateur des villes en laissant de côté ces aspects préoccupants, notamment dans les espaces dits "postcoloniaux»? Plutôt que d'étudier des philosophes ou théoriciens célèbres et bien relayés tel Achille $\mathrm{Mbembe}^{5}$, cet article propose de découvrir et d'analyser les textes d'écrivains moins connus, auteurs de fictions dysphoriques, qui pensent, elles aussi. La réflexion sur la création dans les villes postcoloniales gagnerait sans doute à être à l'écoute de textes littéraires fictionnels qui doutent du potentiel créateur des villes surtout quand ces œuvres sont méconnues, ou inaudibles dans le brouhaha du monde. Notre article prête l'oreille à l'inquiétude sourde et au pessimisme violent qui surgissent de l'écriture de deux auteurs noirs du Sud postcolonial et tâche de comprendre la poétique en jeu dans leurs récits. Nous lirons ensemble deux auteurs qui, à des kilomètres et latitudes de distance, se préoccupent de la terreur et des injustices qui règnent dans les villes postcoloniales, et rapprocherons ainsi le texte d'un auteur martiniquais et d'un poète sud-africain. Ce choix de corpus n'est pas artificiel ou anecdotique. Il résulte d'un engagement critique et d'une attention particulière portée à la littérature postcoloniale des pays dits du Sud. Si la littérature générale et comparée est un effort de réflexion éthique et humaniste comme l'affirment Edward Said ou DanielHenri Pageaux (Gély 2014: 490), l'exercice comparatiste s'enrichit de lectures de productions d'espaces éloignés culturellement et géographiquement, car celles-ci permettent un fécond travail de "défamiliarisation" et peuvent aider à contrer les logiques d'invisibilisation (Lavocat 2012).

Encore peu connus internationalement même s'ils jouissent d'une certaine reconnaissance locale, le Martiniquais Alfred Alexandre et le Sud-Africain Sello K. Duiker réfléchissent à la création et aux villes postcoloniales à travers une écriture qui fait la part belle au tragique et au mystique. Thirteen Cents de Sello K. Duiker $(2000)^{6}$ et Les Villes assassines d'Alfred Alexandre (2011) sont des romans perturbants des villes de bord de mer, Le Cap et Fort-de-France, où des narrateurs personnages (l'un enfant, l'autre déjà homme) tentent de survivre à la jungle urbaine et sont attirés par l'expérience mystique. Michel de Certeau définit le mystique comme :

[...] celui ou celle qui ne peut s'arrêter de marcher et qui, avec la certitude de ce qui lui manque, sait de chaque lieu et de chaque objet que ce n'est pas ça, qu'on ne peut résider ici, ni se contenter de cela. Le désir crée un excès. Il excède, passe et perd les lieux. Il fait aller plus loin, ailleurs. Il n'habite nulle part... (1982 : 411) 
Cet article expliquera pourquoi et comment les personnages de Sello K. Duiker et d'Alfred Alexandre nous poussent à dépasser les apparences et à considérer la ville postcoloniale sous son jour le plus secret et obscur. Dans ces deux récits, le mystique se mêle au tragique, pour s'opposer à l'idée d'une création possible dans la ville : au contraire, les personnages expriment le désir d'annihilation de la ville et suggèrent l'idée de sa nécessaire destruction. Les intuitions et fulgurances du poète haïtien Frankétienne annonceront les étapes de notre raisonnement, ce que nous justifierons au cours de notre étude, même si nous demandons d'emblée au lecteur d'accepter de faire résonner ces voix et d'associer ces imaginaires pour étayer l'analyse et la discussion.

\section{Le choix fictionnel de voix subalternes : dévoiler l'envers de la ville}

Comment parler, sans complaisance, sans épanchements lyriques, d'une aventure tragique qui nous touche de si près? La servitude commence par l'hypothèque des désirs déclenchant une cascade d'inhibitions alimentées par la peur de franchir les frontières artificielles ou naturelles. (Frankétienne [1979] 2010 : 15)

6 Les fictions de Sello K. Duiker et d'Alfred Alexandre révèlent moins une ville " muse " qu'une ville "piège ", où les narrateurs sont comme des prisonniers, effrayés à l'idée de franchir les frontières prescrites par d'autres. Sans faire le jeu du populisme ou du misérabilisme (Passeron et Grignon 1989), il faut expliquer l'importance du choix d'un narrateur « subalterne » dans ces deux romans.

7 Par l'adoption d'un point de vue interne, Sello K. Duiker et Alfred Alexandre font partager aux lecteurs la vision de personnages ordinairement relégués à l'invisibilité, au silence, ou rendus tabous du fait de leurs expériences. Thirteen Cents et Les Villes assassines sont des récits à la première personne assumés par des narrateurs en situation d'infériorité que l'on peut qualifier de «subalternes » et qui finiront rebelles. Azure, le héros de Thirteen Cents, est un jeune Sud-Africain noir âgé de treize ans, qui a fui Johannesburg après avoir découvert chez lui le corps de ses parents assassinés. Il habite sous un pont près de Green Point, souffre de la faim, et se prostitue sur la plage. Il essaie d'échapper aux délinquants qui sévissent dans la ville du Cap mais il lui est difficile de passer inaperçu à cause de ses yeux bleus. Ce détail constitue peut-être un clin d'œil intertextuel au roman de Toni Morrison, The Bluest Eye, dont l'héroïne est une enfant noire et pauvre de Lorain dans l'Ohio, qui croit qu'elle a les yeux bleus après avoir été victime d'inceste. Évane, le héros du roman d'Alfred Alexandre n'est pas un enfant, mais un jeune homme sans emploi d'un quartier populaire situé entre le port de commerce de Fort-de-France et l'une des zones occupées par la SARA7. Coincé entre les rues aux noms fictifs et symboliques de "Fièvre ", «Sans-Retour» et "Veille-aux-Morts", il est amoureux de Winona, liée à Slack, le malfrat du coin. Mais il se préserve de la ville qu'il observe crûment en s'échappant à moto «vers le nord, le matin très tôt, le long des routes qui montent vers la mer " (Alexandre 2011:17).

8 Ces personnages «subalternes" ne sont pas égaux: Azure est plus démuni qu'Évane puisque ce n'est qu'un enfant et qu'il est sans famille et sans abri. Évane est capable de décrypter la réalité comme un jeune adulte et de se protéger. Azure apparaît en revanche comme une victime hyperbolique à cause de tout ce qu'il endure et du statut réifié de son corps, constamment violé. Le regard innocent et naif d'Azure est un procédé énonciatif et narratif qui permet de dénoncer pathétiquement et ironiquement la réalité tout en 
respectant un certain réalisme, tandis que la parole d'Évane, plus travaillée et critique, désigne explicitement les dysfonctionnements de la société. Le «je» dans Les Villes assassines peut se hisser jusqu'à la représentation d'un " nous ", tandis que, dans Thirteen Cents, la solitude d'Azure est irrévocable. Ces héros, aussi différents soient-ils, partagent pourtant les caractères tragiques de la victime sacrificielle, témoin de la violence de la ville et de la société tout entière. En outre, tous deux sont de sexe masculin, incapables de rendre compte de l'expérience des femmes qui les entourent. S'il est vrai que la femme subalterne, est encore "plus profondément dans l'ombre " que l'homme subalterne (Spivak 1988 : 287), les auteurs de nos romans n'ont pas essayé de les faire parler en les muant en narratrices.

Le choix de personnages subalternes dans ces romans des années 2000 est à resituer dans un contexte de réflexion postcolonial en littérature et en sciences sociales sur le silence des sans-voix. Gayatri Spivak dans son célèbre essai « Can the subaltern speak? » reprend la notion de «subalterne » à Antonio Gramsci et à ses Cahiers de prison pour interroger le statut épistémologique au sein des sciences sociales de celui qui, selon elle, n'est "pas simplement opprimé » mais véritablement "sans identité » au point de constituer un « espace de différence » extérieur à tout «impérialisme culturel » (Spivak 1988, 1992 et 2014). Le subalterne peut-il parler de son expérience, et si oui, peut-il être entendu ? La philosophe qui est aussi professeur de lettres répondait par la négative à cette double question, sans ignorer que la littérature est un lieu où peut se briser le silence et où peut être créée une écoute ${ }^{8}$. Les écrivains, sans se soucier forcément des débats des études postcoloniales, se sont depuis longtemps confrontés au problème du silence des sansvoix. Ainsi, Aimé Césaire promettait que «[s]a bouche sera la bouche des malheurs qui n'ont point de bouche» (Césaire [1939] 1983: 22). André Brink concevait l'écrivain comme celui qui doit « tenter infatigablement de dire ce qui ne peut pas être dit » (Brink 1998 : 21). Et pour Toni Morrison, centrer son premier roman The Bluest Eye sur Pecola, «le membre le plus délicat de la société : un enfant, le membre le plus vulnérable : une fillette » sans faire de celle-ci la narratrice, c'était respecter la voix inaudible de la fillette et « sculpter un silence tout en le brisant » (Morrison [1970] 2007 : 13).

Le silence, dans nos récits, est brisé par les héros mêmes. Ils décrivent dans une langue crue et poétique l'envers de la ville et la « vie à l'envers » subie. « Les villes qui fument le crack n'aiment pas qu'on dise qu'elles sont belles. La nuit quand elles allument leurs chandelleries minables sous la pluie, elles ont les yeux qui se rincent le sang, en mille morceaux de miroirs, dans les flaques d'eau » déclare Évane (Alexandre 2011: 11). Les images puissantes de miroir brisé et de sang métaphorisent une ville où sens et identité sont perdus, supplantés par l'étourdissement du sexe, des danses et des drogues. Évane dénonce les hommes politiques, les "officiels", qui, pour mieux "cloîtrer [leurs] rébellions» (Ibid.: 14), laissent les narcotrafiquants "saupoudrer» le quartier. Aussi franc mais plus naïf, Azure explique qu'il est livré à lui-même au Cap :

Je vis seul. Les rues de Sea Point sont ma maison. Mais je suis presque un homme, j'ai bientôt treize ans. Ce qui veut dire que je sais où trouver de la nourriture qui n'a pas été trop visitée par les fourmis ou les mouches à Camps Bay ou Clifton. (Duiker [2000] 2010:11)

Être adulte dans la ville se résume pour Azure à survivre.

11 Ces deux romans montrent la vérité cachée des villes, métonymies des sociétés de leur pays. Ils ne sont pourtant pas de la veine des « roman[s]-trottoir » que l'écrivain congolais Sony Labou Tansi disait « imbattable[s]» parce que «[q]uand le peuple parle, la parole prend sa mesure exacte. Elle tourne en folie et dévaste tout » (Labou Tansi 1988:143, cité 
par Garnier 1999 : 156). En effet, nos héros subalternes ne sont pas « le peuple » tant ils sont à part et sans véritable identité. Bien plus, Thirteen Cents et Les Villes assassines récusent le carnavalesque et le grotesque qui grimacent un sourire. Leurs narrateurs, de par leur marginalité tragique, ne rusent pas avec la violence urbaine mais la fuient et la condamnent. Si, comme l'a noté Achille Mbembe, "grotesque et obscène font partie de l'identité propre des régimes de domination en postcolonie", Azure et Évane n'investissent pas le carnavalesque comme lieu de refuge et moyen de résistance. Leur parole porte au contraire la dénonciation de ce que Mbembe appelle la «zombification mutuelle des dominants et de ceux qu'ils sont censés dominer» (Mbembe $2000: 143$ ). Entre désir de fuite et vœu de destruction, Azure et Évane choisiront une confrontation extrême avec la ville, leur espace clos de vie, comme s'ils étaient conscients que la « connivence [...] bloque dans l'impouvoir » (Ibid.).

\section{Le rêve interdit : fuir la ville-piège et ses sortilèges}

Toutes leurs promesses puent le mensonge. Ils nous ont laissés choir dans la boue. La nuit, nous dormons sous les ponts, sur les trottoirs. Le jour, nous errons à travers les rues de la basse ville, en furetant dans les dépotoirs, aux abords des marchés publics, condamnés à reprendre le même calvaire, les mains vides, les lèvres sèches, la bouche amère, les tripes encordées par la faim. (Frankétienne 2010 : 17)

12 Le sentiment paradoxal d'abandon et de surveillance, la quête de sens et la faim caractérisent Thirteen Cents et Les Villes assassines, même si, dans ce dernier roman, la nourriture est moins un manque qu'une arme d'assujettissement. Slack séduit les adolescentes dont il fera commerce en les invitant chez lui et en les nourrissant. Azure, dont le nom fait écho à celui d'Azaro, le héros du roman du Nigérian Ben Okri intitulé $L a$ Route de la faim (1991), cherche partout à manger y compris chez les hommes à qui il vend son corps. Par la suite, il sera torturé en étant affamé par la bande de Gérald. Les expériences vécues par ces personnages donnent à voir non pas la "ville du colonisé » décrite par Fanon dans Les Damnés de la terre (2002: 42-43) mais un espace postcolonial double où se fréquentent privilégiés et déclassés, et où les puissants ne sont pas plus libres que les opprimés. En ce sens, la description négative de la ville pointe, par-delà le problème des inégalités, une liberté illusoire, à l'échelle de toute la société.

13 Évane insiste sur la complaisance des politiques et des journalistes «qui aimaient de temps en temps venir [leur] chatouiller une caméra sous les narines, histoire qu'[ils] déballonne[nt] au reste du pays comment on s'arrangeait pour se contenter, sans jamais une révolte, d'une existence autant gavée de solitude et de misère " (Alexandre 2011: 26-27). À l'instar des citadins curieux du corps ou de l'expérience de vie d'Azure, des Martiniquais aisés en quête de "pure délivrance " (Ibid.: 27) viennent s'étourdir en soirée, au contact des plus pauvres. La complicité et l'impuissance de la société sudafricaine face aux enfants des rues sont révélées par plusieurs épisodes dans Thirteen Cents - Que penser de ces adolescents blancs qui veulent payer pour connaître «la culture urbaine » d'Azure et de ses amis qualifiés de «survivants » (Duiker [2000] 2010:41), et que dire de ces bons pères de famille qui profitent d'Azure pour quelques rands? Le roman déconstruit l'idée de criminalité juvénile et incrimine les adultes qui entourent Azure'. Par exemple, quand le malfrat Richard emmène Azure à l'hôpital après l'avoir battu et raconte au médecin qu'il a subi une correction de la part d'un commerçant qu'il aurait volé, ce dernier critique aussitôt ces gamins dont « on ne sait pas quoi faire » (Ibid. : 68). Or, le roman dévoile que l'enfant des rues est une proie et que la ville n'est pas son 
terrain de jeu mais un espace piégé. Azure nous révèle, en même temps qu'il les découvre, les règles absurdes qui régissent le monde des adultes: l'argent, l'hypocrisie, le sexe, la lâcheté. Il nous permet aussi de comprendre que les personnages qu'il croise ne sont finalement pas plus libres que lui, qu'il s'agisse des adolescents en quête de sensations fortes, des délinquants violents ou des clients homosexuels ${ }^{10}$ qui se cachent derrière une vie de famille rangée.

Meg Samuelson remarque que "si la vue des personnages de Duiker se baladant au-delà des frontières physiques et sociales assignées peut suggérer le potentiel libératoire des villes, à l'intérieur de celles-ci, les effroyables expériences de maltraitance qu'ils subissent nous rappellent néanmoins les réalités de l'espace urbain d'aujourd'hui " (Samuelson 2007 : 255). Ce qui apparaît en effet dans le parcours d'Azure et d'Évane à travers la jungle urbaine c'est ce siège invisible subi par les habitants et provoqué par les rumeurs, la surveillance, l'interdiction de rêver et la peur. L'anthropologue Arjun Appadurai ne décrit pas autrement ce qu'il appelle la « géographie de la colère » quand il explique qu'elle naît d'« une carte mentale où la guerre, la sécurité, le crime et la terreur se superposent progressivement à la géographie du commerce, des transports, du travail et de la consommation " (Appadurai [2006] 2009 : 143). La terreur au quotidien que vivent Azure et Évane est caractéristique d'un terrorisme intérieur qui fait des habitants des quartiers les plus défavorisés des villes, les témoins et complices d'une guerre souterraine. Appadurai souligne la complexité d'une telle « géographie » lorsqu'il observe que :

[d]ans tous les cas, la géographie de la colère n'est pas une simple carte d'action et de réaction, de minoritisation et de résistance, de hiérarchie d'espace et de site, de séquences nettes de cause et d'effets. Ces géographies sont plutôt le résultat spatial d'interactions complexes entre des événements lointains et des craintes proches, entre de vieilles histoires et de nouvelles provocations, entre des frontières réécrites et des ordres non écrits. (Ibid. : 144-145)

Le fonctionnement spatial trouble de la ville, engendré par la collusion « des événements lointains et des craintes proches ", " des frontières réécrites et des ordres non écrits ", est révélé par nos récits lorsqu'ils mettent en scène des résurgences du passé historique. Évane ressasse l'échec des rébellions des sans-voix comme s'il s'agissait d'un mal ancestral ou d'une malédiction de l'île Martinique. Azure se trouve quant à lui pourchassé par Gérald, le malfrat qui se croit blanc, parce que ce dernier s'est vexé qu'Azure l'ait confondu avec son ami noir Sealy. Qu'il s'agisse de la haine née des identifications raciales incertaines ou de la soumission anonyme aux forts, la ville est présentée dans ces textes comme un espace de danger et de peur où dominent des logiques de magie noire. Les puissants sont effectivement perçus comme dotés de pouvoirs surnaturels. Vincent conseille à Azure d'être discret sinon il sera traqué par les chefs de gangs qui peuvent le suivre grâce à leurs valets transformés en pigeons. L'alcool et la drogue apparaissent aux yeux d'Évane comme les ingrédients quasi magiques qu'utilisent les gangsters et les politiques pour envoûter ${ }^{11}$. Ce thème apparaît également dans Thirteen Cents, quand Azure apprend que sa drogue aurait été trafiquée par Gérald pour le manipuler. Les «géographies de la colère " coïncident donc avec des espaces de croyances en des « ordres sorciers » (Simone 2004).

La perception de la ville de nos héros est magique comme malgré eux, ce qui témoigne de manière réaliste de l'expérience des villes dans les postcolonies. En effet, si l'espace investi par la pensée surnaturelle est souvent rural comme l'ont montré, entre autres, récemment les travaux du couple Comaroff (2010), il est aussi urbain - l'un et l'autre 
étant finalement difficilement dissociables. AbdouMaliq Simone remarque à propos des jeunes Sud-Africains citadins, majoritairement défavorisés, que :

[p]rivés de responsabilités et de certitudes structurantes, les lieux [qu'ils habitent] et les déplacements et actions qu'ils entreprennent deviennent des cas de géographies disjointes - c'est-à-dire subsumés à des ordres mystiques, souterrains ou sorciers, à des univers prophétiques ou apocalyptiques, à des mythes ultralocalisés qui capturent l'allégeance de larges corps sociaux ou qui réinventent quotidiennement des routines qui n'ont à peu près aucun lien avec le reste. (Simone $2004: 7)$

17 Ce constat peut valoir pour les Antillais de même condition. Le besoin d'opposer " quelque contre-réalité » à " un futur indiscernable et [à] une vie incessante de misère » passe par le recours à « diverses formes de spectralité » (Ibid.), ce que donnent à voir nos romans.

La pensée magique y est montrée en rapport avec une situation à la fois métaphorique et réaliste d'esclavage. Slack fait tatouer un « $\mathrm{S}$ » sur le corps des filles qui travaillent pour lui et fait disparaître celles-ci à sa guise, tuées, vendues, ou envoyées dans les hôtels du Sud de l'île selon les rumeurs. Les gangsters du Cap agissent en propriétaires d'esclaves sans pitié vis-à-vis de ceux qu'ils dominent, tel le malheureux Azure, d'ailleurs rebaptisé «Blue» par Gérald. La ville postcoloniale apparait comme le nouveau domaine des esclavagistes dans ces récits, et c'est pourquoi l'univers des années 2000 qu'ils décrivent au Cap et à Fort-de-France ressemble de façon si troublante à la campagne zombifiée haïtienne soumise au terrible Saintil, dans le roman de Frankétienne, Les Affres d'un défi. Ce roman, d'abord écrit en 1975 en créole sous le titre Dézafi puis réécrit par l'auteur en français en 1979, donne à voir l'enfermement, la violence et les rêves brisés du village de Bois-Neuf à la ville de Port-au-Prince. Il peut être lu à plus d'un titre comme un texte déployant de multiples problématiques reprises par les romans contemporains du tragique urbain tant ceux-ci sont aux prises avec la peur et les réalités obscures de la «zombification» et de l'« idéocide» (Appadurai [2006] 2009) liées aux géographies de la colère.

\section{De la fuite à la destruction : violence, mystique et féminin}

Nous apprenons le métier de vivre en marchant à tâtons sur une corde tendue dans le vide, le cœur en équilibre sur la tête, une lampe nue dans nos mains tremblantes. (Frankétienne [1979] 2010 : 157-158)

Nos héros se trouvent dans des villes hantées par les spectres des discriminations passées et sont piégés dans un Fort-de-France à l'image du pays pour Évane, et dans un Cap hyperbolique des vices de la nation pour Azure. La question qui se pose à eux semble être alors celle posée par Achille Mbembe: "Comment vivre quand il est interdit d'être vivant ? C'est-à-dire comment peut-on vivre tout en allant à la mort? et vivre dans la mort?» (Mbembe 2000 : 258). La première réponse de nos personnages est la fuite, ou pour reprendre les mots de Frankétienne, « apprendre le métier de vivre en marchant à tâtons sur une corde tendue dans le vide ». Azure et Évane avancent dans l'obscurité et l'inconnu, et «marronnent » afin d'éviter un « devenir-zombi » dans la ville. Pour l'un comme pour l'autre, le « marronnage » est arpentage et découverte de nouveaux espaces. Azure est un marcheur, plus proche de la figure du mystique sous cet aspect qu'Évane, pour qui fuite rime avec vitesse. Le premier est mû par un véritable martyr, le second est 
pris dans une course folle, quand il parcourt le Nord à moto puis quand il court littéralement à sa perte en se précipitant dans la ville pour tuer Slack d'un coup de revolver. Malgré ces différences, ces marronnages tournent tragiquement à l'obsession de la destruction dans chacun de ces récits.

Évane qui rêve de «sortir de l'avenue » s'oppose à Slack, convaincu « qu'en dehors de la zone autorisée, entre la rue Fièvre et la rue Sans Retour, jamais il n'y aurait pour des gens comme [eux], d'espace où prendre ses aises » (Alexandre $2011: 33$ ). Il se contente d'abord d'un marronnage sans conséquence dans le Nord puis commet une transgression plus grande quand il emmène loin de la ville et des « vies mortes " (Ibid. : 56) Winona, favorite de Slack. La géographie symbolique du roman est partagée entre un Nord idéalisé, ouvert sur la mer des possibles, la ville de Fort-de-France, basse, mauvaise, reliée à un port emblématique de tous les trafics et à un Sud touristique, pétri de vices, et l'archipel, troisième espace, tissé d'inconnu, où un refuge serait possible. Winona a grandi en croyant que sa mère, Éva, arrivée clandestinement en Martinique, est repartie vivre dans l'île inconnue au nom rêveur d'« Éden Ouest ». Mais il s'agit d'un mensonge : la mère, tuée par le père Doppy, repose dans le jardin de la maison où la rejoindra Winona, assassinée par les sbires de Slack. La fuite par l'amour et la contemplation de la nature, équivalentes à une aventure mystique, s'avèrent impossibles dans le roman. Évane dont le prénom même est une répétition vaine du nom de la mère perdue, répète l'échec de la fuite de celle-ci : il ne peut que proposer des «rêves bloqués » et des « chemins qui ne [mènent] nulle part » (103), parce que, comme il le dit lui-même, «[i]l y a des villes qui assassinent la vie. Il y a des villes qui assassinent l'amour » (55).

21 Le féminin est le ressort du tragique dans Les Villes assassines et l'amour, la voie éphémère de la courte expérience mystique du héros. Sa retraite amoureuse dans le Nord avec Winona lui fait éprouver un sentiment de complétude loin des corruptions de la ville, mais la mort de la jeune femme et les confidences du père de celle-ci le précipitent dans l'action meurtrière. Évane ne châtie pas Doppy, mari meurtrier et père aux désirs incestueux, parce que « la vie lui a mis trop de coups, ça lui a zombifié la cervelle », dit-il (128). Il tue en revanche les malfrats Vénaton et Slack pour détruire la gangrène urbaine et agir en héros :

Il fallait que quelqu'un tombe pour effacer, ne serait-ce que quelques secondes, les haut-le-cœur que notre condition, à nous autres reclus, levait dans la poitrine indifférente des salauds bien comme il faut là-bas nichés dans les versants les plus adoucis du pays. (131-132)

La révolte suicidaire d'Évane n'en fait pas un mystique mais un héros tragique piégé par l'enfer de la ville et de la société qu'elle représente. La destruction est symbolique, saisie au vol par un excipit abrupt montrant le héros lancé à l'assaut de Slack.

La fin de Thirteen Cents, plus ambiguë, en dissonance avec le réalisme affiché du début, a été peu commentée si ce n'est par Sam Raditlhalo qui déclare qu'elle paraît «forcée » et " défai[t] la beauté du texte qui précède » (Raditlhaho $2004:$ 75). Pourtant, si on lit cette fin comme une expérience de métamorphose mystique, elle paraît moins être un décrochage que la suite logique d'un roman dont la poétique réaliste et tragique s'efforce jusqu'au bout d'interpeller le lecteur sur la question du sens. En effet, comme l'explique Xavier Garnier, « avec le roman, la magie devient le point sensible d'une interrogation sur le réel et son complément, l'irréel » (Garnier 1999 : 5). Non seulement l'épisode " sorcier » d'Azure est emblématique du caractère paradoxal de bien des romans réalistes qui relatent les expériences les plus extrêmes des sans-voix ${ }^{12}$, mais il rattache Thirteen Cents à 
une longue tradition d'écriture qui incorpore le magique dans le réalisme (Garnier 1999). Pour comprendre ce qui se passe exactement à la fin de ce récit, la notion de « roman réaliste irrationnel » proposée par Xavier Garnier (ibid.) et la définition du fantastique selon Tzvetan Todorov (1970) sont utiles, la prise en compte de la question du mystique (Certeau 1982) est indispensable.

Le « roman réaliste irrationnel » suppose « la complicité totale du narrateur avec la vision magico-religieuse du monde » (Garnier 1999:16) et le fait que « tout doit y être motivé » ( Ibid. : 20). Précisément, Azure, le narrateur, croit aux « ordres sorciers » et son expérience finale, qui paraît irréelle, est préparée et motivée par plusieurs événements traumatiques dans le récit. En effet, c'est son vécu de "martyr » qui annonce l'expérience mystique d'Azure et qui le pousse à passer du " petit marronnage " au bord de la ville, près de la plage, vers Sea Point, au "grand marronnage" vers la montagne de la Table. C'est également ce martyr qui le transforme en être obsédé par la destruction. Le rapt dont il est victime est décisif : maltraité plusieurs jours, il est ensuite violé collectivement. Quand il revient à la ville, le malfrat Gérald, à l'origine de ses souffrances, lui fait subir une sorte de rituel et lui parle de ses parents qu'il déclare avoir tués. Confus et infirme, Azure cherche à fuir la ville mais trahi par son amie Liesel, il en est réduit à se prostituer. Il entame sa première ascension de la montagne quand Gérald le pourchasse à nouveau, et sa transformation, remarquée par les autres personnages après son rapt, se confirme. Azure ne veut plus fuir la ville mais la détruire, ce qu'il se répète obstinément en fumant du cannabis. Il dit son attirance pour la montagne, sa haine et son mépris du Cap : "Quand je regarde derrière moi, je vois la ville maintenant paisible. Elle s'étend faible, au-dessous de moi. Je crache. Je vais t'écraser, je me dis, et j'avance » (Duiker [2000] 2010 : 171).

Le récit distille alors des indices propices à une interprétation mystique. Azure rencontre Oscar, un homme blanc, mi-passant, mi-ermite, marginalisé par son homosexualité, et dont il prédit que la montagne le rendra fou. Mais cet Oscar n'est-il pas le double du héros, par-delà les différences de race, de classe, ou de sexualité ? Azure ne prédit-il pas sa propre folie? La perte d'identité du héros fait de son corps « l'objet d'une marche qui va, comme tout pèlerinage, vers un site marqué par une disparition » (Certeau 1982 : 108). La nuit dans une grotte auprès d'un feu, il entrevoit l'origine du monde à travers un personnage féminin qui lui apparaît en rêve: une petite femme callipyge nommée "Saartjie », en qui le lecteur reconnaît Saartjie Baartman, « la Vénus hottentote ${ }^{13}$ ». Cette femme emblématique de la disparition des origines pleure et se plaint de la ville mais elle active aussi un récit mythique selon lequel Gérald serait son mari et un monstre qui mange ses propres enfants, et Azure son fils. Quand Azure retourne au Cap et apprend la mort de Gérald, il passe quelque temps avec Sealy qui s'est présenté à lui comme l'« ange de la destruction » et lui a affirmé que lui, Azure, est l'héritier de Gérald et que son destin est de détruire la ville. Mais Sealy est finalement embarqué par les policiers, et Azure retourne dans la grotte de la montagne, d'où il croit entendre et voir la destruction de la ville sous les vagues et dans les flammes. Le récit s'achève alors qu'il s'adonne à une danse hypnotique autour du feu.

Azure ne remet pas en question ce qui lui arrive, même si le lecteur peut s'interroger sur la réalité de ce qui est décrit. Thirteen Cents serait un « roman réaliste irrationnel » qui, dans sa réception, provoque le sentiment du fantastique chez le lecteur, c'est-à-dire selon Tzvetan Todorov ${ }^{14}$, cette " hésitation éprouvée par un être qui ne connait que les lois naturelles, face à un événement en apparence surnaturel » (Todorov $1970: 29$ ). On hésite 
entre croire Azure, adhérer à l'incroyable surgissant dans la fiction et considérer que le héros est frappé de folie. Le récit active en même temps ces deux lectures concurrentes, l'une et l'autre aussi tragique. Il a tant insisté sur l'aspect maléfique de la ville et la prédiction de sa destruction que l'explication surnaturelle apocalyptique semble possible. Cependant la lecture qui verrait dans cet excipit un message messianique reste douteuse. Comment Azure peut-il être un sauveur s'il est annoncé comme l'héritier de Gérald ? Et comment savoir si le bien triomphe du mal dans cette destruction finale chaotique dont, finalement, notre héros est écarté ? Ces questions restent dangereusement sans réponse. Par ailleurs, le récit a tant insisté sur les croyances et les drogues que l'explication rationnelle par le délire semble valoir aussi. L'indécision est la richesse de ce final violent. Elle n'escamote en rien l'expérience mystique que vit Azure, au contraire. La rencontre avec le féminin originel en rêve, la confusion des sens et la danse sont au cœur de cette expérience, dont Michel de Certeau a dit qu'elle avait " souvent l'allure d'un poème qu'on "entend" comme on entre dans une danse. Le corps est "informé" (il reçoit la forme) de ce qui lui arrive [...] bien avant que l'intelligence en ait connaissance » $(1982: 408)$.

\section{Conclusion}

Ainsi, dans Thirteen Cents et Les Villes assassines, la ville postcoloniale est racontée par des héros « subalternes » comme un univers où se tissent les « géographies de la violence » et où se déploie une compréhension du monde qui dépend des "ordres sorciers ». La poétique tragique de la ville est dès lors étroitement dépendante des thèmes de la quête mystique et du désir de fuir, même s'il s'agit d'une mystique sans Dieu et d'une fuite impossible ${ }^{15}$. Azure et Évane se transforment en allant au bout de la logique de privation qui les caractérise, ce qu'attestent leurs ultima verba. Le premier déclare : «J'ai vu le cœur de l'obscurité. J'ai vu le négrier de l'obscurité et c'est un fou furieux. Je connais ses secrets. Je sais ce qu'il fait pendant notre sommeil. Ma mère est morte. Mon père est mort » (Duiker [2000] 2010 : 230), le second lance : « Ni patrie. Ni Église. Ni amour pour personne» (Alexandre 2011: 135). Le dépouillement dans la destruction en fait des victimes sacrificielles, bien que le récit s'arrête avant leur mort, finalement indécidable. Les récits abattent froidement le mythe d'un renouveau social et culturel déployé grâce aux villes, qu'il s'agisse de la «ville capitale ${ }^{16}$ » ou du Cap, métropole attractive d'une nation « arc-en-ciel » que l'ont dit réconciliée. Réfléchir au potentiel créateur des villes postcoloniales pourrait être l'occasion de méditer sur ces fictions contemporaines qui se présentent comme de funestes témoignages de l'expérience urbaine et qui interrogent la possibilité d'une utopie et d'une victoire du sens. En effet, le lien avec l'expérience mystique, la répétition de la perte, l'expérience de la folie et le sacrifice n'ôtent pas aux romans de Sello K. Duiker et d'Alfred Alexandre leur force réaliste, mais « ouvre[nt] » au contraire davantage «la fiction à la question, profondément littéraire, du non-sens » (Garnier 2008). 


\section{BIBLIOGRAPHIE}

Abramson, Helen, 2009, New Spaces, New Identities: The City in Post-Apartheid South African Fiction, Mémoire de Master d'Anglais soutenu à l'Université d'Amsterdam, [En ligne], http://dare.uva.nl/ cgi/arno/show.cgi?fid=147188, consulté le 10 janvier 2015 .

Appadurai, Arjun, [1996] 2001, Après le colonialisme : les conséquences culturelles de la globalisation [ Modernity at Large: Cultural Dimensions of Globalization], préface de Marc Abélès, traduit de l'anglais par Françoise Bouillot, Paris, Payot.

Appadurai, Arjun, [2006] 2009, Géographie de la colère : la violence à l'âge de la globalisation [Fear of Small Numbers: an Essay on the Geography of Anger], traduit de l'anglais par Françoise Bouillot, Paris, Payot \& Rivages.

Appiah, Kwame Anthony, [2006] 2008, Pour un nouveau cosmopolitisme [Cosmopolitanism: Ethics in a World of Strangers], traduit de l'anglais par Agnès Botz, Paris, Odile Jacob.

Augé, Marc, 1992, Non-lieux. Introduction à une anthropologie de la surmodernité, Paris, Le Seuil.

Awondo, Patrick, 2014, « L'afropolitanisme en débat », Politique africaine, $\mathrm{n}^{\circ}$ 136, p. 105-119.

Brink, André, 1998, «Interrogating Silence: New Possibilities Faced by South African Literature », dans D. Attridge et R. Jolly (dir.), Writing South Africa: Literature, Apartheid, and Democracy, 1970-1995 , Cambridge, Cambridge University Press, p. 14-28.

Bessière, Irène, 1973, Le Récit fantastique, la poétique de l'incertain, Paris, Larousse.

Brival, Hervé, « La SARA : un mastodonte fier de ses 40 années », France-Antilles, vendredi

21 janvier 2011, [En ligne], http://www.martinique.franceantilles.fr/actualite/economie/la-saraun-mastodonte-fier-de-ses-40-annees-21-01-2011-99495.php, consulté le 20 juin 2015.

Certeau, Michel de, 1982, La Fable mystique : XVI ${ }^{e}-X V I I^{e}$ siècle, Paris, Gallimard.

Comaroff, Jean et Comaroff, John, 2010, Zombies et frontières à l'ère néolibérale. Le cas de l'Afrique du Sud postapartheid, préface et traduction française de Jérôme David, Paris, Les Prairies ordinaires.

Davis, Mike, 2006, Planet of Slums, Londres, Verso books.

Demissie, Fassil, 2007, « Imperial Legacies and Postcolonial Predicaments: An Introduction », African Identities, vol. 5, $\mathrm{n}^{\circ}$ 2, p. 155-165.

Fanon, Frantz, [1961] 2002, Les Damnés de la terre, préface d'Alice Cherki et postface de Mohammed Harbi, Paris, La Découverte.

Garnier, Xavier, 1999, La Magie dans le roman africain, Paris, PUF.

Garnier, Xavier, 2008, résumé de La magie dans le roman africain, [En ligne], halshs-00289215, consulté le 10 décembre 2014.

Gély, Véronique, 2014, "Comparaison et comparatismes : regards actuels », Revue de littérature comparée $\mathrm{n}^{\circ} 352$, p. 489-502.

Harpin, Tina, Inceste, race et histoire : fictions et contre-fictions de pouvoir dans les romans sud-africains et états-uniens des XX et XXI siècles, Thèse de doctorat, Université Paris 13, décembre 2013. 
Johns, Timothy, 2010, « The Price of Pleasure: Sello Duiker's Thirteen Cents and the Economics of Homosexuality in South Africa », dans H. Nabasuta Mugambi et T. Jita Allan (dir.), Masculinities in African literary and cultural texts, avant-propos d'A. Kalu et postface de S. Gikandi, Banbury, Ayebia Clarke.

Lavocat, Françoise, 2012, « Le comparatisme comme herméneutique de la défamiliarisation », Vox Poetica, [En ligne], http://www.vox-poetica.org/t/articles/lavocat2012.html.

Mbembe, Achille, 2000, De la postcolonie : essai sur l'imagination politique dans l'Afrique contemporaine, Paris, Karthala.

Mbembe, Achille, 2004, avec Nuttall Sarah, « Writing the World from an African Metropolis », Public Culture, vol. 16, $\mathrm{n}^{\circ}$ 3, p. 347-372.

Mbembe, Achille, 2005, « Afropolitanisme », Africultures, [En ligne], http:// www.africultures.com/php/?nav=article $\&$ no $=4248$.

Mbembe, Achille, 2010, Sortir de la grande nuit : essai sur l'Afrique décolonisée, Paris, La Découverte.

Mbembe, Achille, 2013, Critique de la raison nègre, Paris, La Découverte.

Passeron, Jean-Claude et Grignon, Claude, 1989, Le Savant et le Populaire. Misérabilisme et populisme en sociologie et en littérature, Paris, Seuil.

Pélis, Yoann, 2005, Les Nouveaux Défis de l'énergie à la Martinique, Paris, PubliBook, Société des écrivains.

Raditlhalo, Sam, 2004, « “A victory of sorts”: All Thirteen Cents and Bitter, Too », dans I. Visser et H. van den Heuvel-Disler (dir.), Family Fictions in English, The Family in Contemporary Postcolonial Literatures in English, Actes du colloque tenu à l'université de Groningen, aux Pays-Bas, du 17 au 19 novembre 2004, p. 65-80, [En ligne], http://www.rug.nl/research/globalisation-studiesgroningen/cds/publications/familyfictions.pdf, consulté le 8 mars 2010.

Samuelson, Meg, 2007, « The City Beyond the Border: The Urban Worlds of Duiker, Mpe and Vera ", African identities, vol. 5, $\mathrm{n}^{\circ}$ 2, p. 247-260.

Simone, Abdou Maliq, 2004, For the City yet to Come: Changing African Life in Four Cities, Durham, Duke University Press.

Spivak, Gayatri Chakravorty, 1988, « Can the Subaltern Speak? », dans C. Nelson et L. Grossberg (dir.), Marxism and the Interpretation of Culture, Urbana (IL), University of Illinois Press, p. 271-313.

Spivak, Gayatri Chakravorty, 1992, avec De Kock Leon, «Interview With Gayatri Chakravorty Spivak: New Nation Writers Conference in South Africa », ARIEL: A Review of International English Literature, vol. 23, $\mathrm{n}^{\circ} 3$, p. 29-47.

Spivak, Gayatri Chakravorty, 2014, « On n'est pas subalterne parce qu'on le ressent ! », entretien publié sur le site de Philo Mag, http://www.philomag.com/les-idees/entretiens/gayatri-spivakon-nest-pas-subalterne-parce-quon-le-ressent-2082, consulté le 18 décembre 2014.

Todorov, Tzvetan, 1970, Introduction à la littérature fantastique, Paris, Seuil.

\section{Corpus}

Alexandre, Alfred, 2011, Les Villes assassines, Paris, Écriture.

Duiker, K. Sello, [2000] 2002, Thirteen cents, Le Cap, Ink inc.

Duiker, K. Sello, 2010, 13 cents traduit de l'anglais par Laura Derajinski, Paris, Yago. 


\section{Corpus secondaire}

Césaire, Aimé, [1939] 1983, Cahier d'un retour au pays natal, Paris, Présence Africaine.

Frankétienne, [1979] 2010, Les Affres d'un défi, La Roque-d'Anthéron, Vents d'ailleurs.

Laferrière, Dany, [1985] 2002, Le Cri des oiseaux fous, Paris, Le serpent à plumes.

Morrison, Toni, [1970] 2007, The Bluest Eye, New York, Vintage International Edition.

Okri, Ben, [1991] 1994, La Route de la faim [The Famished Road], traduit de l'anglais par Aline Weill, Paris, Julliard.

\section{NOTES}

1. L'afropolitanisme s'oppose à l'afrocentrisme condamné par Mbembe dans Critique de la raison nègre (Mbembe 2013 : 255). Il s'agit plutôt d'« une stylistique, une esthétique et une certaine poétique du monde », or, cette «manière d'être au monde [...] refuse, par principe, toute forme d'identité victimaire - ce qui ne signifie pas qu'elle n'est pas consciente des injustices et de la violence que la loi du monde a infligé à ce continent et à ses gens. C'est également une prise de position politique et culturelle par rapport à la nation, à la race et à la question de la différence en général » (Mbembe 2005).

2. "Simon Gikandi définit l'afropolitanisme comme "une nouvelle phénoménologie de l'Africanité" (new phenomenology of Africanness) ", comme le remarque Patrick Awondo (2014: 113).

3. L'APELA organisait en septembre 2015 à l'université de Bourgogne, un colloque intitulé «Panafricanisme, cosmopolitisme et "afropolitanisme" dans les littératures africaines». Les propos cités sont tirés de l'appel à contribution publié par cette association en vue du colloque (voir le document en ligne sur le site de cette association: http://www.apela.fr/wp-content/ uploads/2010/01/afropolitanisme_APELA.pdf.pdf).

4. Ce contraste des expériences et des écritures de la ville a été mis en avant dans le colloque «Représenter la ville sud-africaine / Representing South African Cities » tenu les 27 et 28 novembre 2013 à l'université Paris Ouest Nanterre La Défense et organisé par les centres CAS de Toulouse, CREA et Centre Espaces/Écritures de Nanterre en collaboration avec le Gordon Institute for Performing and Creative Arts du Cap.

5. Le 2 mai 2016, Achille Mbembe était invité par Alain Mabanckou au Collège de France à participer au colloque «Penser et écrire l'Afrique». Son intervention, mise en ligne sur le site du Collège de France sous le titre «Afropolitanisme et afrofuturisme », ne reprend pourtant aucun de ces deux termes : https://www.youtube.com/watch?v=Pe60QeqLqb4.

6. J'utilise la traduction de Laura Derajinski pour citer le roman dans cet article. Les autres traductions de l'anglais sont de moi sauf mention contraire.

7. La SARA est la Société anonyme de raffinerie des Antilles créée sous l'impulsion du général de Gaulle en 1969, pour " permettre aux Antilles-Guyane d'avoir une indépendance énergétique » (Brival 2011). En réalité, cette société reste la marque de la « dépendance structurelle » des DOM dans l'approvisionnement en produits pétroliers (Pélis 2005: 40). En outre, accusée de faire partie du groupe des "profiteurs ", la SARA a été au cœur de la tourmente lors de la grève de 2009 en Guadeloupe et en Martinique, puisque l'une des principales revendications des grévistes était la baisse des prix du carburant.

8. Cette notion d'écoute est importante dans les derniers travaux de la philosophe, notamment dans sa réflexion sur l'enseignement de la littérature. 
9. Voir sur ce point Timothy Johns (2010).

10. Loin de se résumer à la question de la prostitution, l'homosexualité est abordée de façon complexe dans ce roman qui refuse tout simplisme et manichéisme (Johns 2010).

11. C'est là une idée commune aux légendes urbaines concernant le crack, notamment aux Antilles françaises. Surnommé « diab'la » (le diable, en créole), le crack, en Guadeloupe et en Martinique, a la réputation d'être utilisée comme arme de nuisance à la fois dans les rapports interpersonnels et à un niveau politique. Certaines rumeurs affirment en effet qu'on aurait laissé cette drogue s'infiltrer aux Antilles pour briser les ferments de révolte et faire échouer les projets indépendantistes. Voir Un caillou et des hommes, le documentaire diffusé sur France ô et tourné en 2014 par les écrivaines Fabienne et Véronique Kanor.

12. Sur le lien entre réalisme, grotesque et récits fictionnels de subalternes, voir Tina Harpin (2013).

13. Cette femme "Khoikoï » (le mot « hottentot » est perçu comme péjoratif) figure une histoire ancienne et complexe de migrations, de performance, de racisme et d'humiliation. Pour les descendants de ce peuple autochtone de l'Afrique australe, elle est devenue emblématique de l'oubli qu'ils ont subi dans l'histoire, mais elle est aussi un symbole national, comme l'attestent les célébrations officielles qui ont accompagné le rapatriement de son corps en Afrique du Sud en 2002.

14. Pour une réflexion critique sur cette définition, il est intéressant de lire Irène Bessière (1973). 15. En ce sens, le cheminement de ces héros est inverse à celui du narrateur du Cri des oiseaux fous de Dany Laferrière qui a pu fuir la ville de Port-au-Prince. Son dépouillement final augure alors un véritable renouveau, comme en témoignent ses mots : "Une ville nouvelle à connaître par cœur. Sans guide. Ni dieu. Les dieux ne m'accompagneront pas. L'ancien monde ne pourra m'être d'aucun secours " ([1985] $2002: 344)$. Il est intéressant de noter que pas plus que leurs héros, Alfred Alexandre et Sello K. Duiker n'ont choisi l'exil à la différence de Dany Laferrière. Les limites du sens et de la création se sont malheureusement imposées à l'écrivain sud-africain qui a choisi de s'exiler de la vie même, le 19 janvier 2005.

16. Cette expression a fleuri à la fin des années 1990 en Martinique pour évoquer Fort-de-France dans certains discours politiques et dans les médias locaux. L'appellation exacte de la ville serait «chef-lieu » puisque la Martinique n'est pas un État indépendant doté d'une capitale, mais un département français.

\section{RÉSUMÉS}

Si la ville en mouvement postcoloniale est porteuse d'espoirs pour de nombreux écrivains et penseurs, elle reste aussi la muse plus sombre d'un questionnement existentiel sur le sens. Cet article examine les enjeux de la poétique tragique de la ville à l'œuvre dans les romans Thirteen Cents de Sello K. Duiker (2000) et Les Villes assassines d'Alfred Alexandre (2011) en expliquant la part d'expérience mystique logée au cœur de la description de la violence dans ces récits assumés par des personnages marginalisés et subalternes, qui ne savent plus que dire leur volonté de détruire.

If the postcolonial city characterized by its movement provides hope for many writers and thinkers, it also persists as the gloomy muse that inspires an existential questioning of the meaning of life. In this article, I examine the issues at stake in the tragic poetics of the city in 
motion in the novels Thirteen Cents by Sello K. Duiker (2000) and Les Villes assassines by Alfred Alexandre (2011) and explain the element of mysticism at the heart of the depiction of violence embodied by menial and marginalized characters who have no choice but to voice their will to destroy.

INDEX

Mots-clés : ville, subalterne, postcolonial, mystique, magie

Keywords : city, subaltern, postcolonial, mystical, magic

\section{AUTEUR}

TINA HARPIN

Université de Guyane 